\title{
Механизм генерирования донорно-акцепторных пар при сильном легировании $n$-ZrNiSn акцепторной примесью Ga
}

\author{
(C) B.A. Ромака ${ }^{1,2}$, P.-F. Rogl ${ }^{3}$, D. Frushart ${ }^{4}$, D. Kaczorowski ${ }^{5}$ \\ ${ }^{1}$ Институт прикладных проблем механики и математики им. Я. Пидстрыгача Национальной академии наук Украины, \\ 79060 Львов, Украина \\ ${ }^{2}$ Национальный университет „Львовская политехника“, \\ 79013 Львов, Украина \\ ${ }^{3}$ Институт ффизической химии Венского университета, \\ А-1090 Вена, Австрия \\ ${ }^{4}$ Институт Нееля Национального центра научных исследований, \\ 38042 Гренобль, Франция \\ ${ }^{5}$ Институт низких температур и структурных исследований им. В. Тшебетовского Польской академии наук, \\ 50-950 Вроцлав, Польша \\ E-mail: vromaka@polynet.Iviv.ua
}

(Получена 31 мая 2017 г. Принята к печати 31 мая 2017 г.)

\begin{abstract}
Установлена природа механизма одновременного генерирования донорно-акцепторных пар при сильном легировании интерметаллического полупроводника $n-\mathrm{ZrNiSn}$ акцепторной примесью $\mathrm{Ga}$. Найдено такое пространственное расположение атомов в кристаллической решетке $\mathrm{ZrNiSn}_{1-x} \mathrm{Ga}_{x}$, когда скорость движения уровня Ферми $\varepsilon_{\mathrm{F}}$, полученная из расчетов распределения плотности электронных состояний, совпадает с экспериментально установленной из зависимостей $\ln \rho(1 / T)$. Показано, что при занятии примесным атомом $\mathrm{Ga}\left(4 s^{2} 4 p^{1}\right)$ позиции $4 b$ атомов $\mathrm{Sn}\left(5 s^{2} 5 p^{2}\right)$ одновременно генерируются как структурные дефекты акцепторной природы, так и донорной в виде вакансий в позиции $4 b$. Обсуждение результатов ведется в рамках модели сильно легированного и компенсированного полупроводника Шкловского-Эфроса.
\end{abstract}

DOI: 10.21883/FTP.2018.03.45614.8573

\section{1. Введение}

Термоэлектрические материалы, полученные путем сильного легирования интерметаллических полупроводников $n$-TiNiSn, $n$-ZrNiSn и $n$-HfNiSn акцепторными и(или) донорными примесями [1], показывают высокие значения термоэлектрической добротности $Z$ и обладают высокой эффективностью преобразования тепловой энергии в электрическую. Так, в термоэлектрических материалах $\mathrm{Ti}_{1-x-y} \mathrm{Zr}_{x} \mathrm{Hf}_{y} \mathrm{NiSn}(0 \leq x \leq 1 ; 0 \leq y \leq 1)$ [2] и $\mathrm{Ti}_{0.5} \mathrm{Zr}_{0.25} \mathrm{Hf}_{0.25} \mathrm{Ti}_{0.5} \mathrm{NiSn}_{0.998} \mathrm{Sb}_{0.002}$ [3] значения ZT при температуре $T=400 \mathrm{~K}$ равны $\sim 1.2$, что отвечает лучшим показателям материалов на основе теллуридов, клатратов и скутерудитов. Простота синтеза, отсутствие токсичных или иных вредных для окружающей среды элементов, а также стабильность характеристик в диапазоне $4.2-1000 \mathrm{~K}$ делают интерметаллические полупроводники широко исследуемыми в научных центрах.

Широкое внедрение этих материалов тормозят не полностью изученные процессы трансформации кристаллической и электронной структур при оптимизации характеристик путем легирования базового полупроводника, что сопровождается непрогнозируемым генерированием структурных дефектов, влияющих на его электронную структуру. Так, при сильном легировании $n$-ZrNiSn акцепторными примесями $\mathrm{M}=\mathrm{Cr}, \mathrm{Mn}, \mathrm{Fe}$, $\mathrm{Co}$, введенными в полупроводник путем замещения $\mathrm{Ni}$, появляются доноры неизвестной природы [3-6]. Более логичным было генерирование в $\mathrm{ZrNi}_{1-x} \mathrm{M}_{x} \mathrm{Sn}$ только акцепторов, поскольку число $3 d$-электронов $\mathrm{Ni}$ больше, чем в $\mathrm{Cr}, \mathrm{Mn}, \mathrm{Fe}$ и $\mathrm{Co}$. Однако кинетические исследования выявили появление доноров, концентрация которых растет вместе с концентрацией примеси.

В термоэлектрическом материале $\mathrm{TiNiSn}_{1-x} \mathrm{Ga}_{x}$, полученном легированием $n$-TiNiSn атомами $\mathrm{Ga}\left(4 s^{2} 4 p^{1}\right)$ путем замещения $\mathrm{Sn}\left(5 s^{2} 5 p^{2}\right)$, также установлено одновременное генерирование как акцепторов (у Ga меньше p-электронов, чем у $\mathrm{Sn}$ ), так и доноров, концентрация которых росла с увеличением содержания $\mathrm{Ga}[7,8]$.

Как известно, термоэлектрические материалы на основе интерметаллических полупроводников синтезируют путем сплавления шихты исходных компонентов с последующим охлаждением расплава [1], а это, по определению Н. Мотта [9], один из способов получения аморфных твердых тел. Кроме того, оптимизация характеристик материала путем легирования полупроводника дополнительно генерирует дефекты донорной и(или) акцепторной природы, а в запрещенной зоне появляются энергетические состояния, которые и определяют его свойства. Таким образом, по способу получения исследуемые термоэлектрические материалы являются сильно легированными и сильно компенсированными полупроводниками (СЛСКП) [10].

Б. Шкловский и А. Эфрос показали, что наличие в полупроводнике значительного числа заряженных дефектов, расположение которых носит флуктуационный характер, радикально изменяет его электронную структуру и приводит к флуктуации потенциального рельефа 


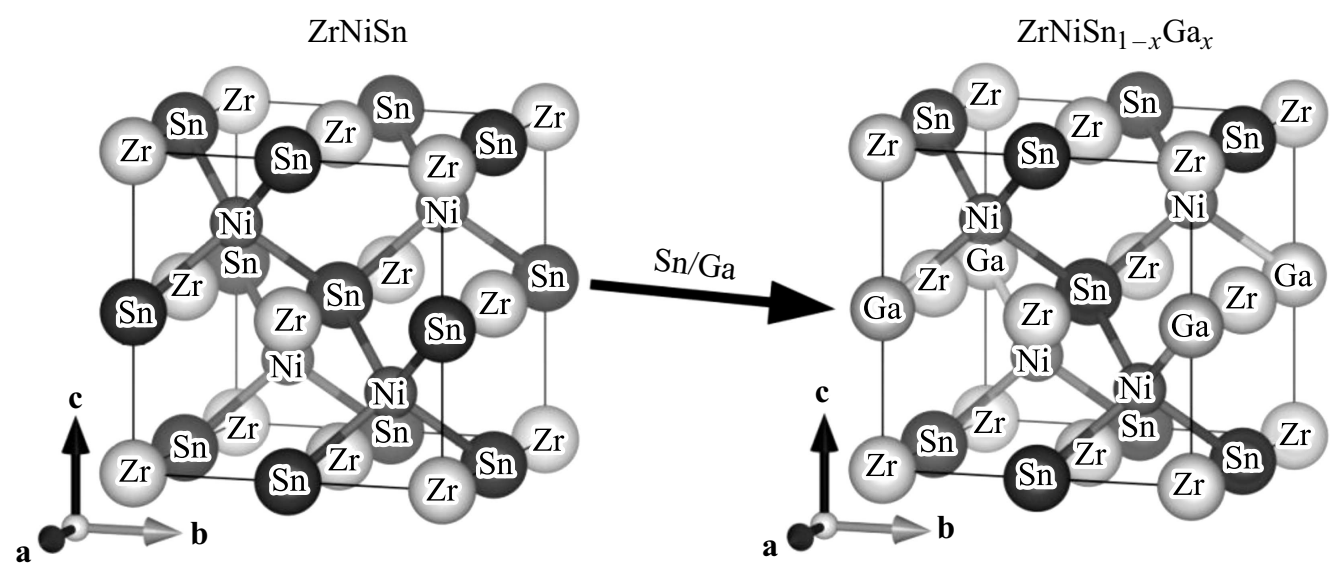

Рис. 1. Модель трансформации кристаллической структуры $\mathrm{ZrNiSn}$ в $\mathrm{ZrNiSn}_{1-x} \mathrm{Ga}_{x}$.

и модуляции зон непрерывных энергий [11,12]. При низких температурах сильно легированный полупроводник - неупорядоченная система, которая напоминает аморфные системы. Авторы работы [12] предложили схему полностью компенсированного полупроводника как модель аморфного полупроводника, когда электрон движется не в периодическом поле кристала, а в хаотическом поле дефектов.

Используя подходы из работ $[11,12]$ для анализа неупорядоченных систем, к которым относятся интерметаллические полупроводники [1], далее будет показан механизм одновременного генерирования дефектов акцепторной и донорной природы (донорно-акцепторные пары) в термоэлектрическом материале $\mathrm{ZrNiSn}_{1-x} \mathrm{Ga}_{x}$. Учет механизма при синтезе таких материалов позволит существенно повысить их эффективность в процессе преобразования тепловой энергии в электрическую.

\section{2. Методики исследований}

Образцы были синтезированы в лаборатории Института физической химии Венского университета. Методом рентгеноструктурного анализа (дифрактометр GuinierHuber image plate system, $\mathrm{Cu} K_{\alpha 1}$-излучение) получены значения периодов кристаллической решетки $a(x)$, а при помощи программы Fullprof [13] установлены координаты атомов, тепловые параметры, занятость позиций. Химический и фазовый составы контролировались при помощи сканирующего электронного микроскопа Zeiss Supra 55VP. Стандартные отклонения не превышали $1 \%$. Расчет электронной структуры проводился методом функций Грина (KKR) в приближении когерентного потенциала (CPA) и локальной плотности (LDA) [14], а также в рамках теории функционала плотности DFT. Использовали экспериментальные значения постоянной решетки на $k$-сетке размером $10 \times 10 \times 10$ и тип параметризации обменно-корреляционного потенциала Moruzzi-Janak-Williams [15]. Ширина энергетического окна равна 16 эВ, а точность расчета положения уровня
Ферми $\varepsilon_{F}$ составляла \pm 8 мэВ для 1000 значений энергии. Измерялись температурные и концентрационные зависимости удельного сопротивления $(\rho)$, коэффициента термоэдс $(\alpha)$ относительно меди и магнитной восприимчивости $(\chi)$ образцов $\mathrm{ZrNiSn}_{1-x} \mathrm{Ga}_{x}$ в диапазонах: $T=80-400 \mathrm{~K}, \quad N_{\mathrm{A}}^{\mathrm{Ga}} \approx 1.9 \cdot 10^{20} \mathrm{~cm}^{-3}(x=0.01)$ $-2.9 \cdot 10^{21} \mathrm{~cm}^{-3}(x=0.15) \quad$ и магнитного поля $H \leq 10$ кЭ.

\section{3. Исследование особенностей кристаллической структуры $\mathrm{ZrNiSn}_{1-x} \mathrm{Ga}_{x}$}

Микрозондовый анализ атомов на поверхности образцов $\mathrm{ZrNiSn}_{1-x} \mathrm{Ga}_{x}$ установил их соответствие исходным составам шихты, а рентгеновские фазовый и структурный анализы показали, что дифрактограммы образцов индексируются в структурном типе MgAgAs (пространственная группа $F \overline{4} 3 m$ ) [16] и не содержат следов других фаз (рис. 1).

Термодинамические расчеты в приближении гармонических колебаний в рамках теории функционала плотности DFT показали, что изменение значений свободной энергии $\Delta G(x)$ (потенциал Гельмгольца) проходит через минимум в $x \approx 0.4$ при температуре отжига образцов $T=1073 \mathrm{~K}$ (рис. 2, $a$ ), указывая на предел растворимости $\mathrm{Ga}$, в который попадают исследуемые образцы $\mathrm{ZrNiSn}_{1-x} \mathrm{Ga}_{x}, x=0-0.15$.

В работе [17] показано, что структура $n-\mathrm{ZrNiSn}$ неупорядочена (локальная аморфизация [1]) в результате частичного, до $\sim 1 \%(z \approx 0.01)$, занятия $\mathrm{Ni}\left(3 d^{8} 4 s^{2}\right)$ позиции $4 a$ атомов $\operatorname{Zr}\left(4 d^{2} 5 s^{2}\right)$, что генерирует структурные дефекты донорной природы („априорное легирование“), поскольку у $\mathrm{Ni}$ больше $d$-электронов, а в запрещенной зоне появляются донорные состояния $\varepsilon_{\mathrm{D}}^{1}$. Формула полупроводника преобразуется в $\left(\mathrm{Zr}_{1-z} \mathrm{Ni}_{z}\right) \mathrm{NiSn}$.

Учитывая меньший атомный радиус $\mathrm{Ga}\left(r_{\mathrm{Ga}}=\right.$ $=0.141 \mathrm{Hм})$ по сравнению с $\mathrm{Sn} \quad\left(r_{\mathrm{Sn}}=0.162 \mathrm{HM}\right)$, ожидалось уменьшение значений периода кристал- 

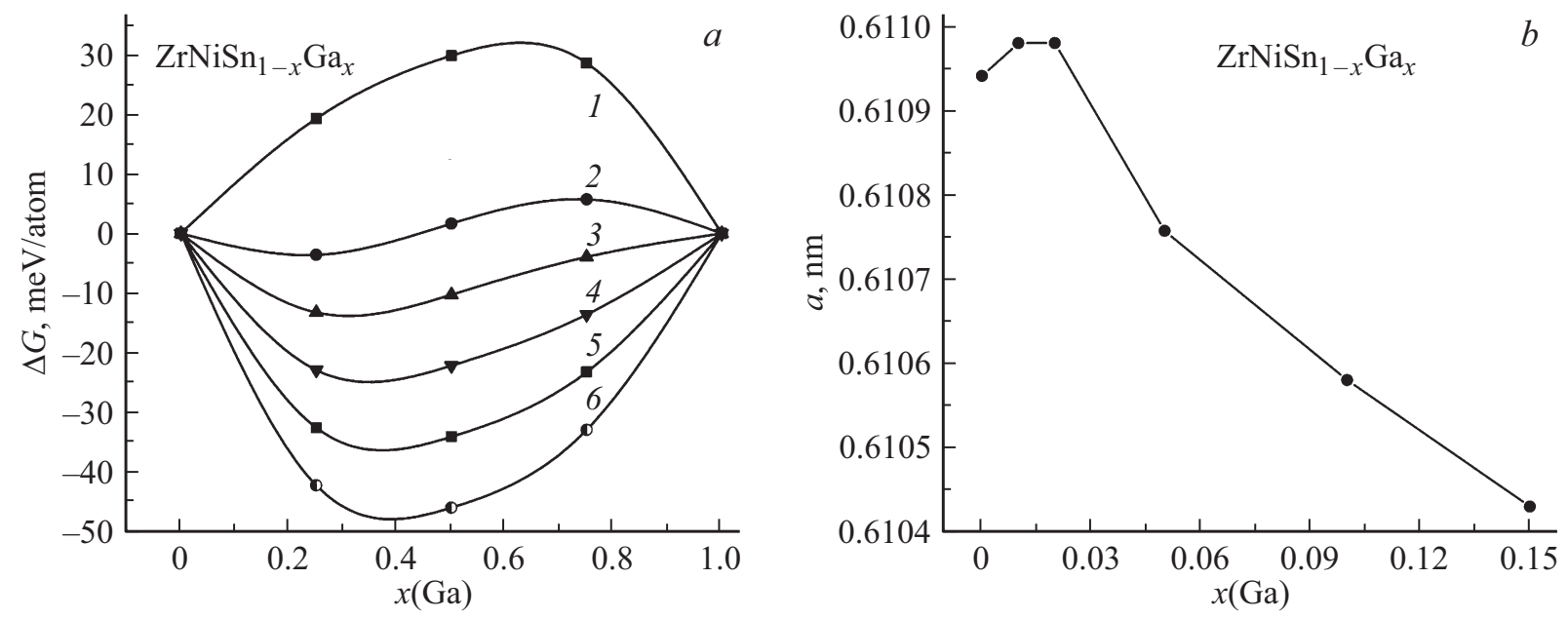

Рис. 2. Изменение значений термодинамического потенциала $\Delta G(x)$ при разных температурах $T, \mathrm{~K}: 1-0,2-473,3-673$, $4-873,5-1073,6-1273(a)$ и периода кристаллической решетки $a(x)(b) \mathrm{ZrNiSn}_{1-x} \mathrm{Ga}_{x}$.

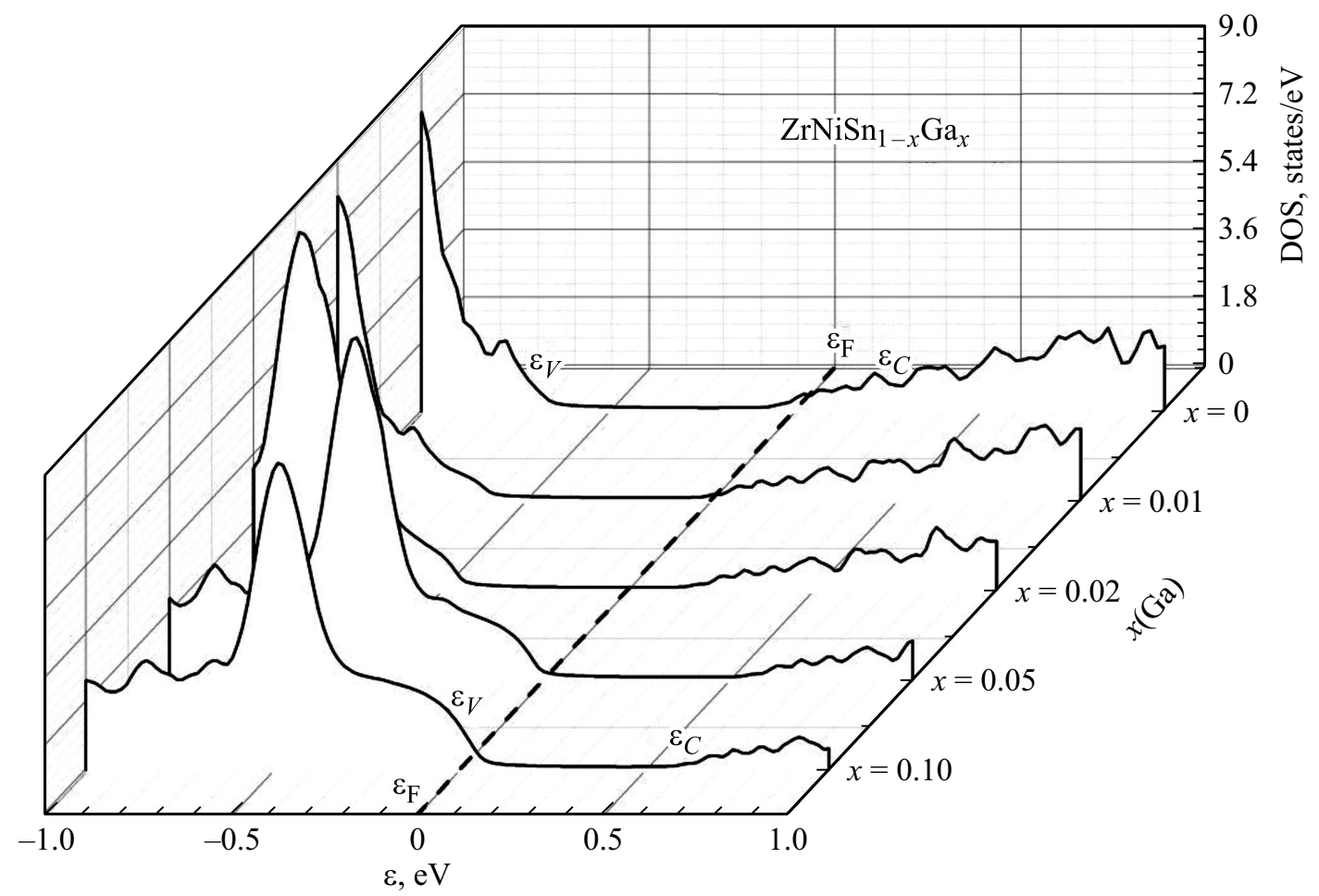

Рис. 3. Расчет распределения плотности электронных состояний $\mathrm{DOS} \mathrm{ZrNiSn}_{1-x} \mathrm{Ga}_{x}$.

лической решетки $a(x) \mathrm{ZrNiSn}_{1-x} \mathrm{Ga}_{x}$. Однако, как видно из рис. $2, b$, изменение $a(x)$ носит немонотонный характер, отражая процессы структурных преобразований, влияющих на электронную структуру и свойства полупроводника. Обращаем внимание на характер изменения $a(x)$ на участке концентраций $0 \leq x \leq 0.03$.

Как показали структурные исследования, на участке концентраций примеси $\mathrm{Ga} 0 \leq x \leq 0.01$ структура $\mathrm{ZrNiSn}_{1-x} \mathrm{Ga}_{x}$ упорядочивается („залечиваются“ дефекты) путем вытеснения небольших атомов $\mathrm{Ni}$ $\left(r_{\mathrm{Ni}}=0.124 \mathrm{Hм}\right)$ с позиции $4 a$ большими атомами $\mathrm{Zr}$
$\left(r_{\mathrm{Zr}}=0.160\right.$ нм $)$, а значения $z \rightarrow 0$. Параллельно происходит замещение в позиции $4 b$ больших атомов Sn на меньшие $\mathrm{Ga}$ (рис. 1). Учитывая, что разница атомных радиусов $\mathrm{Zr}$ и Ni равна $\left(r_{\mathrm{Zr}}-r_{\mathrm{Ni}}\right)=0.036 \mathrm{нм}$, а $\mathrm{Sn}$ и $\mathrm{Ga}\left(r_{\mathrm{Sn}}-r_{\mathrm{Ga}}\right)=0.021 \mathrm{Hм}$, изменение $a(x)$ на участке $0 \leq x \leq 0.01$ будет определять процесс вытеснения $\mathrm{Ni}$ с позиции $4 a$ большими атомами $\mathrm{Zr}$, что вызывает рост зависимости $a(x)$ (рис. $2, b)$. После вытеснения атомов $\mathrm{Ni} \mathrm{c}$ позиции $\mathrm{Zr}$ (упорядочение структуры) уменьшение значений $a(x)$ на участе $0.02 \leq x \leq 0.15$ будет определять заполнение позиции $\mathrm{Sn}(4 b)$ атомами $\mathrm{Ga}$ (рис. $2, b)$. 
Изменения в кристаллической структуре $\mathrm{ZrNiSn}_{1-x} \mathrm{Ga}_{x}$ при ее упорядочении приведут к перестройке электронной структуры. Так, если в $n-\mathrm{ZrNiSn}$ в запрещенной зоне существуют донорные состояния $\varepsilon_{\mathrm{D}}^{1}$, образованные в результате вытеснения $\sim 1 \%$ атомов $\mathrm{Zr}$ атомами Ni [17], то упорядочение структуры (вытеснение $\mathrm{Ni}$ с позиции $\mathrm{Zr}(4 a)$ ) уменьшит число дефектов донорной природы, а донорные состояния $\varepsilon_{\mathrm{D}}^{1}$ исчезнут.

Так как Ga имеет на один $p$-электрон меньше, чем $\mathrm{Sn}$, то замещение $\mathrm{Sn}$ на Ga генерирует в позиции $4 b$ дефекты акцепторной природы, а в запрещенной зоне появятся акцепторные состояния $\varepsilon_{\mathrm{A}}$. Наличие значительного числа акцепторов и доноров изменит электронную структуру $\mathrm{ZrNiSn}_{1-x} \mathrm{Ga}_{x}[11,12]$, что должно проявиться при исследовании кинетических и энергетических характеристик полупроводника.

\section{4. Исследование электронной структуры $\mathrm{ZrNiSn}_{1-x} \mathrm{Ga}_{x}$}

Для прогнозирования поведения уровня Ферми $\varepsilon_{\mathrm{F}}$, ширины запрещенной зоны $\varepsilon_{g}$ и кинетических характеристик $\mathrm{ZrNiSn}_{1-x} \mathrm{Ga}_{x}$ был проведен расчет плотности электронных состояний (DOS). Принимая во внимание результаты структурных исследований, расчет проводился для упорядоченного варианта структуры $\mathrm{ZrNiSn}_{1-x} \mathrm{Ga}_{x}$, т.е. все атомы занимают собственные кристаллографические позиции [16] (рис. 3).

Легирование $n$ - ZrNiSn наименьшей концентрацией Ga увеличивает степень компенсации, а уровень Ферми $\varepsilon_{\mathrm{F}}$ (пунктирная линия) дрейфует от уровня протекания зоны проводимости, на расстоянии $\sim 97.6$ мэВ от которого он находился [1], к середине запрещенной зоны. При больших концентрациях примеси $\mathrm{Ga}$ уровень Ферми $\varepsilon_{\mathrm{F}}$ пересечет середину запрещенной зоны $(x \approx 0.025)$ и будет двигаться к уровню протекания валентной зоны, который пересечет при $x \approx 0.05$ : реализуется переход проводимости диэлектрик-металл (переход Андерсона [9]). Изменится тип проводимости и дырки станут основными носителями тока, а дальнейшее легирование акцепторами $p-\mathrm{ZrNiSn}_{1-x} \mathrm{Ga}_{x}(x \approx 0.04)$ уменьшит степень компенсации полупроводника.

Таким образом, расчет DOS $\mathrm{ZrNiSn}_{1-x} \mathrm{Ga}_{x}$ на основе результатов структурных исследований указывает на линейную зависимость скорости дрейфа уровня Ферми $\varepsilon_{\mathrm{F}}$, изменение типа основных носителей и реализацию перехода проводимости диэлектрик-металл. Результаты исследований кинетических характеристик $\mathrm{ZrNiSn}_{1-x} \mathrm{Ga}_{x}$ покажут степень адекватности результатов расчета реальным процессам в полупроводнике.

\section{5. Исследование кинетических характеристик $\mathrm{ZrNiSn}_{1-x} \mathrm{Ga}_{x}$}

Температурные и концентрационные зависимости удельного сопротивления $\rho$ и коэффициента термо- эдс $\alpha \mathrm{ZrNiSn}_{1-x} \mathrm{Ga}_{x}$ приведены на рис. 4, 5. Зависимости $\ln \rho(1 / T)$ и $\alpha(1 / T)$ (рис. 4) типичные для СЛСКП, а наличие активационных участков указывает на несколько механизмов переноса заряда. Зависимости $\ln \rho(1 / T)$ можно представить при помощи известного выражения [10]:

$$
\rho^{-1}(T)=\rho_{1}^{-1} \exp \left(-\frac{\varepsilon_{1}^{\rho}}{k_{\mathrm{B}} T}\right)+\rho_{3}^{-1}\left(-\frac{\varepsilon_{3}^{\rho}}{k_{\mathrm{B}} T}\right),
$$

где первый высокотемпературный член описывает активацию носителей тока $\varepsilon_{1}^{\rho}$ с уровня Ферми $\varepsilon_{\mathrm{F}}$ на уровень протекания зон непрерывных энергий, а второй, низкотемпературный, - прыжковую проводимость $\varepsilon_{3}^{\rho}$. Температурные зависимости коэффициента термоэдс $\alpha(1 / T) \quad \mathrm{ZrNiSn}_{1-x} \mathrm{Ga}_{x}$ описывает следующая зависимость [9]:

$$
\alpha=\frac{k_{\mathrm{B}}}{e}\left(-\frac{\varepsilon_{i}^{\alpha}}{k_{\mathrm{B}} T}-\gamma+1\right),
$$

где $\gamma$ - параметр, зависящий от механизмов рассеивания. С высокотемпературных участков зависимостей $\alpha(1 / T)$ вычислены значения энергии активации $\varepsilon_{1}^{\alpha}$, которые пропорциональны амплитуде крупномасштабной флуктуации зон непрерывных энергий, а с низкотемпературных - значения энергии активации $\varepsilon_{3}^{\alpha}$, пропорциональные амплитуде модуляции мелкомасштабной флуктуации СЛСКП.

Наличие на всех зависимостях $\ln \rho(1 / T)$ $\mathrm{ZrNiSn}_{1-x} \mathrm{Ga}_{x}$ высокотемпературных активационных участков указывает на расположение уровня Ферми $\varepsilon_{\mathrm{F}}$ в запрещенной зоне. Такое поведение зависимостей $\ln \rho(1 / T)$ не соответствует результатам расчета DOS (рис. 2): прогнозировалось пересечение уровнем Ферми $\varepsilon_{\mathrm{F}}$ уровня протекания валентной зоны при концентрации $\mathrm{Ga} x \approx 0.05$ и металлизация проводимости.

Если предположить, что в $\mathrm{ZrNiSn}_{1-x} \mathrm{Ga}_{x}$, как ожидалось, генерируются только дефекты акцепторной природы, то при концентрации примеси $\mathrm{Ga}$, например, $x=0.15\left(N_{\mathrm{A}}^{\mathrm{Ga}} \approx 3 \cdot 10^{21} \mathrm{~cm}^{-3}\right)$ уровень Ферми $\varepsilon_{\mathrm{F}}$ должен находиться глубоко в валентной зоне. Однако при высоких температурах наблюдаем активацию, что в полупроводнике возможно только при условии одновременного с акцепторами генерирования доноров неизвестного происхождения, которые компенсируют акцепторы, вынудив уровень Ферми $\varepsilon_{\mathrm{F}}$ находиться в запрещенной зоне, отражая степень компенсации $\mathrm{ZrNiSn}_{1-x} \mathrm{Ga}_{x}$.

Исследования кристаллической и электронной структур показали, что замещение атомов $\mathrm{Sn}$ на Ga приведет к генерированию в позиции $4 b$ дефектов акцепторной природы, а в запрещенной зоне - примесных акцепторних состояний $\varepsilon_{\mathrm{A}}$, и дырки станут основными носителями тока. Такое предположение оправданно, так как при концентрации Ga $x>0.01$ число генерированных акцепторних состояний $\varepsilon_{\mathrm{A}}$ уже превышает число доноров с энергией $\varepsilon_{\mathrm{D}}^{1}$ в $n-\mathrm{ZrNiSn}$ (число атомов $\mathrm{Ni}$ в позиции $\operatorname{Zr}(4 a))$. 

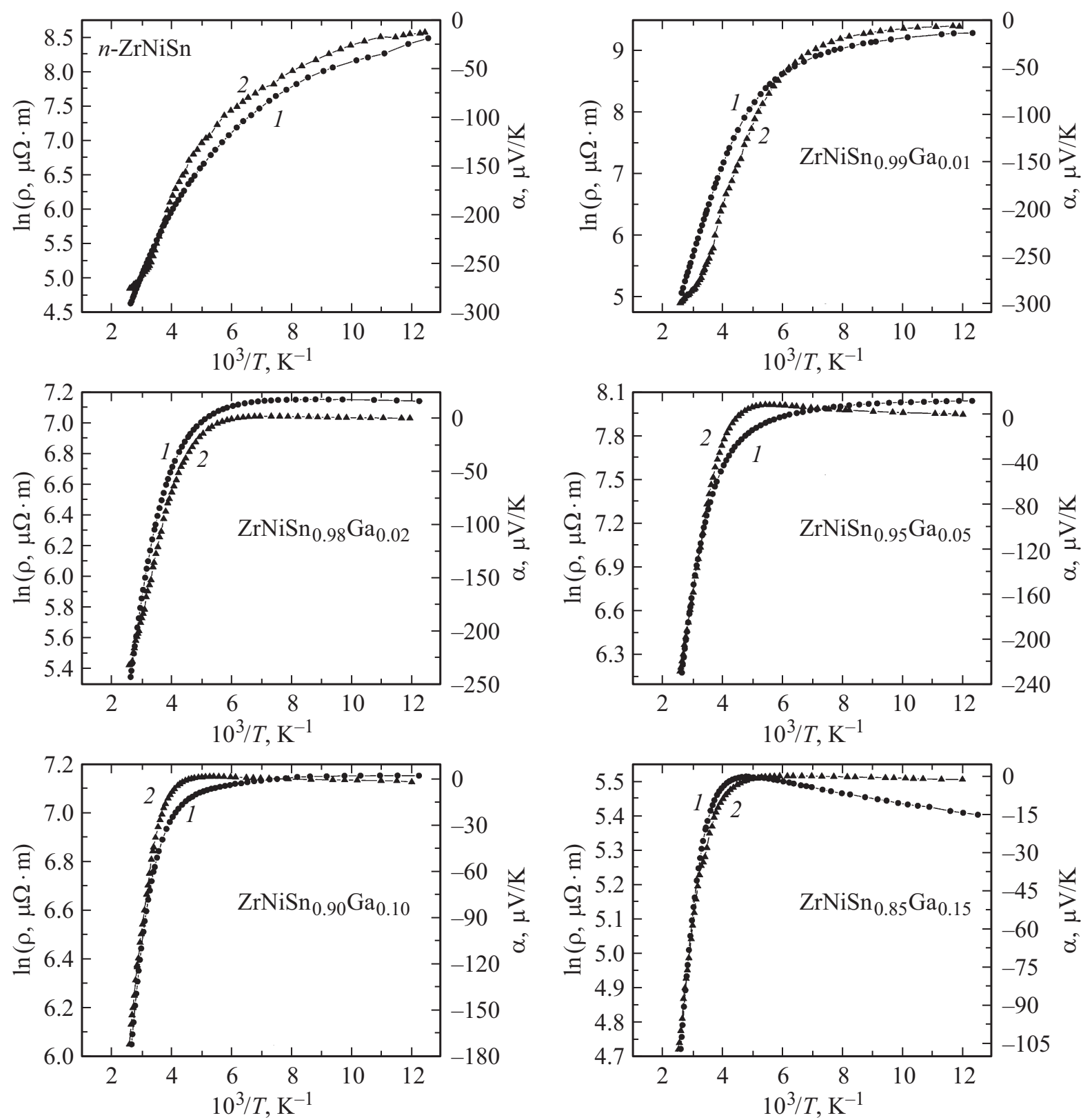

Рис. 4. Температурные зависимости удельного сопротивления $\ln \rho(1 / T)(1)$ и коэффициента термоэдс $\alpha(1 / T)(2) \mathrm{ZrNiSn}_{1-x} \mathrm{Ga}_{x}$.

С другой стороны, если предположить, что в $n$ - $\mathrm{ZrNiSn}$ отсутствуют структурные дефекты донорной природы и полупроводник является собственным (отсутствуют донорные состояния $\left.\varepsilon_{\mathrm{D}}^{1}\right)$, то при легировании полупроводника $\mathrm{Ga}$ значения удельного сопротивления $\rho(x)$ будут уменьшаться при всех температурах и концентрациях из-за появления и увеличения числа свободных дырок при ионизации акцепторов $\varepsilon_{\mathrm{A}}$. Таким образом, знак коэффициента термоэдс будет положительным.

Акцепторная примесь $\mathrm{Ga}$ генерирует структурные дефекты акцепторной природы, что следует из зависимостей изменения удельного сопротивления $\rho(x, T)$ и коэффициента термоэдс $\alpha(x, T)$ (рис. 5). Вна- чале проведем анализ зависимостей $\rho(x)$ и $\alpha(x)$ при температуре $80 \mathrm{~K}$. Так, введение минимальной концентрации $\mathrm{Ga}$ увеличивает значения удельного сопротивления от $\rho(x=0)=4751.1$ мкОм - м до $\rho(x=0.01)=10677.7$ мкОм $\cdot$ м. Так как в образце с $x=0.01$ число акцепторов одного порядка с числом доноров в $n$ - $\mathrm{ZrNiSn}$, он будет сильно легированным и сильно компенсированным.

Рост зависимости $\rho(x)$ на участе концентрации $x=0-0.01$ вызван двумя процесами:

- уменьшение числа донорных состояний $\varepsilon_{\mathrm{D}}^{1}$ при упорядочении структуры $\mathrm{ZrNiSn}_{1-x} \mathrm{Ga}_{x}$, когда атомы $\mathrm{Zr}$ вытесняют с позиции $4 a$ атомы $\mathrm{Ni}$, „залечивая“ структурные дефекты донорной природы; 

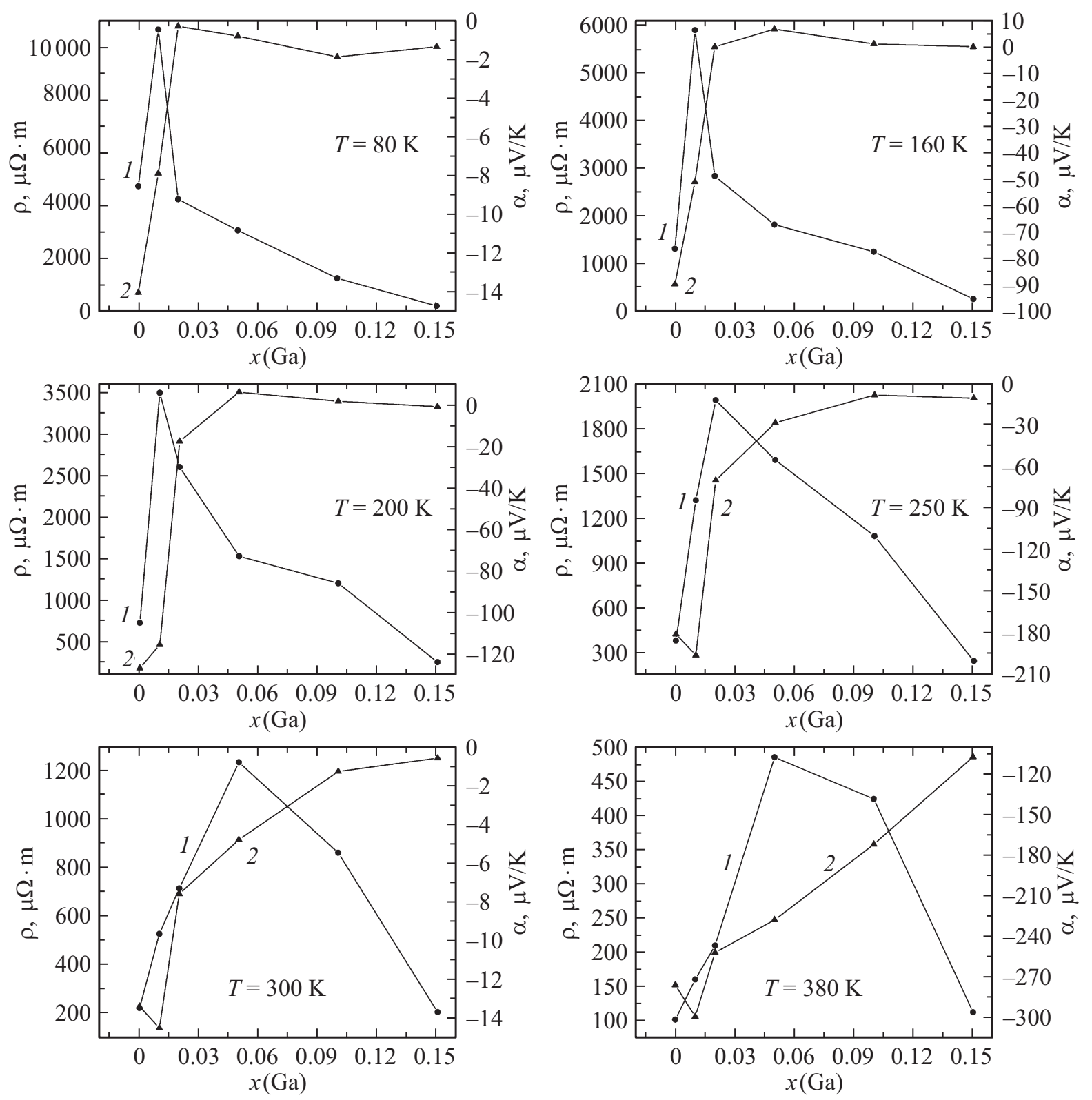

Рис. 5. Изменение значений удельного сопротивления $\rho(x)(1)$ и коэффициента термоэдс $\alpha(x)(2) \mathrm{ZrNiSn}_{1-x} \mathrm{Ga}_{x}$ при разных температурах.

- „вымораживание“ свободных электронов в запрещенную зону на примесные акцепторные состояния $\varepsilon_{\mathrm{A}}$, образованные при замещении Sn на Ga.

Однако при концентрации $x>0.01$ и $T=80 \mathrm{~K}$ значения удельного сопротивления $\rho(x)$ $\mathrm{ZrNiSn}_{1-x} \mathrm{Ga}_{x}$ также стремительно уменьшаются от $\rho(x=0.02)=4255.89$ мкОм $\cdot$ м $\quad$ до $\rho(x=0.05)=$ $=3079.7$ мкОм $\cdot$ м $\quad$ и $\quad \rho(x=0.10)=1275.7$ мкОм $\cdot$ м, указывая на увеличение числа свободных носителей тока. Максимум на зависимости $\rho(x)$ в полупроводнике $n$-типа при легировании акцепторной примесью Ga отражает уравновешивание механизмов токопереноса. Исследуем тип и природу происхождения носителей тока, которые вызывают спад зависимости $\rho(x)$ (рис. 5).
Как следует из рис. 4, 5, при $T=80 \mathrm{~K}$ знак коэффициента термоэдс $\mathrm{ZrNiSn}_{1-x} \mathrm{Ga}_{x}$ отрицательный для всех концентраций примеси (электроны - основные носители тока). И это при том, что концентрация дефектов акцепторной природы в образце, например, $\mathrm{ZrNiSn}_{1-x} \mathrm{Ga}_{x} \quad(x=0.10)$, на порядок превышает концентрацию доноров в $n-\mathrm{ZrNiSn}$. Это возможно только при условии такой глубины залегания акцепторных состояний, что температура $80 \mathrm{~K}$ является недостаточной для их ионизации (преодоления дыркой энергетического барьера между уровнем протекания валентной зоны и уровнем $\left.\varepsilon_{\mathrm{A}}\right)$.

С ростом температуры $(T>80 \mathrm{~K})$ зависимость $\rho(x)$ изменяется: при $T=160 \mathrm{~K}$ и $x \approx 0.06$ появляется сту- 


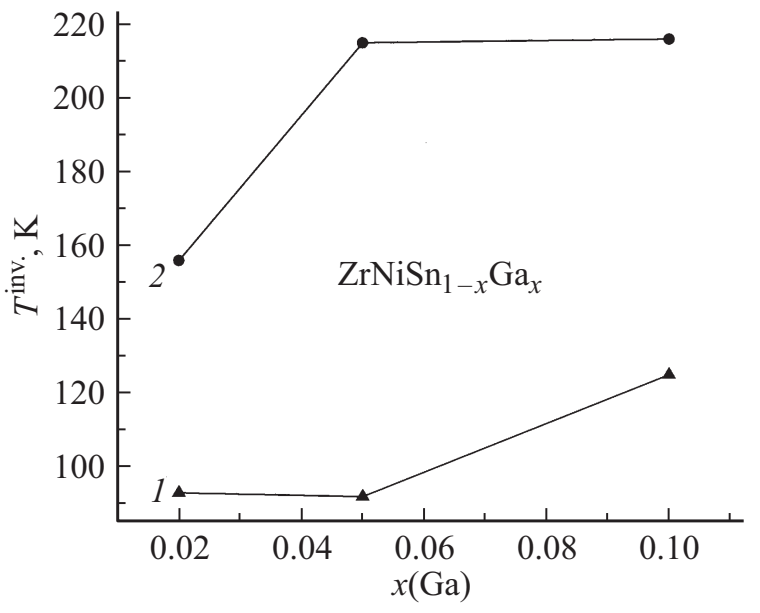

Рис. 6. Изменение значений температуры инверсии $T^{\text {inv }}$ знака коэффициента термоэдс $\mathrm{ZrNiSn}_{1-x} \mathrm{Ga}_{x}: 1-T_{1}^{\text {inv }}$ (изменение знака с отрицательного на положительный); $2-T_{2}^{\text {inv }}$ (изменение знака с положительного на отрицательный).

пенька, которая перерастает в экстремум $(T=300 \mathrm{~K})$ и с ростом температуры $(T=380 \mathrm{~K})$ смещается в область больших концентраций, отображая изменения в электронной структуре. При этом пропадает максимум при $x \approx 0.01$.

Таким образом, при небольших концентрациях Ga максимум на зависимости $\rho(x)$ при $x \approx 0.01$ (рис. 5) обусловлен наличием донорных состояний $\varepsilon_{\mathrm{D}}^{1}(\mathrm{Ni}$ в позиции $\mathrm{Zr}$ ). При концентрации $\mathrm{Ga}$, которая близка к концентрации доноров в $n$-ZrNiSn $(x \approx 0.01)$, значение $\rho(x)$ максимально, а полупроводник становится сильно компенсированным. Так как значения коэффициента термоэдс при $T=80 \mathrm{~K}$ отрицательные для всех концентраций примеси, эта температура недостаточна для полной ионизации акцепторов.

При концентрациях примеси $x \approx 0.02$, когда число акцепторов превышает число доноров в $n-\mathrm{ZrNiSn}$, при повышении температуры до $T=T_{1}^{\text {inv }}$ коэффици- ент термоэдс меняет знак с отрицательного на положительный (рис. 6) в результате ионизации акцепторов $\varepsilon_{\mathrm{A}}$. Однако при дальнейшем увеличении температуры до $T=T_{2}^{\text {inv }}$ в образцах $\mathrm{ZrNiSn}_{1-x} \mathrm{Ga}_{x}, x \approx 0.02$ и $x \approx 0.05,0.10$, при $T \approx 156 \mathrm{~K}$ и $T \approx 216 \mathrm{~K}$ соответственно знак коэффициента термоэдс опять становится отрицательным (рис. 6). При этом концентрация генерированных акцепторов в $\mathrm{ZrNiSn}_{1-x} \mathrm{Ga}_{x}$, $x>0.01$, намного превышает число доноров $\varepsilon_{\mathrm{D}}^{1}$ в $n$-ZrNiSn. Такое поведение коэффициента термоэдс возможно только при условии, что в полупроводнике наряду с акцепторными состояниями генерируются донорные состояния $\varepsilon_{\mathrm{D}}^{2}$, энергетические уровни которых более глубокие, чем $\varepsilon_{\mathrm{D}}^{1}$, и для их ионизации нужны большие энергии. Таким образом, в полупроводнике $\mathrm{ZrNiSn}_{1-x} \mathrm{Ga}_{x}$ одновременно генерируются донорноакцепторные пары.

К таким же результатам приводит анализ поведения $\rho(x) \mathrm{ZrNiSn}_{1-x} \mathrm{Ga}_{x}$ при разных температурах. Так как экстремум $\rho(x)$ отражает уравновешивание конкурирующих процессов в электронной структуре полупроводника, то при повышении температуры ликвидация максимума $\rho(x)$ при небольших концентрациях примеси $\mathrm{Ga}(x \approx 0.01)$ и появление нового экстремума при $x \approx 0.06$ указывают на существование донорных состояний $\varepsilon_{\mathrm{D}}^{2}$, глубина залегания которых больше, чем $\varepsilon_{\mathrm{D}}^{1}$. Действительно, при $x \geq 0.02$ и температурах $T=<T_{2}^{\text {inv }}$, значения которых недостаточны для ионизации донора $\varepsilon_{\mathrm{D}}^{2}$, концентрация акцепторов превышает концентрацию доноров и знак коэффициента термоэдс положительный. При больших температурах $\left(T \geq T_{2}^{\text {inv }}\right)$ начинается ионизация доноров $\varepsilon_{\mathrm{D}}^{2}$, резко увеличивается число свободных электронов, они становятся основными носителями тока, на что указывают отрицательные значения коэффициента термоэдс.

С другой стороны, чем большее число акцепторов генерируется в $\mathrm{ZrNiSn}_{1-x} \mathrm{Ga}_{x}$, тем необходимы большие энергии (большие температуры), чтобы число ионизированных доноров $\varepsilon_{\mathrm{D}}^{2}$ превосходило число акцепторов $\varepsilon_{\mathrm{A}}$.
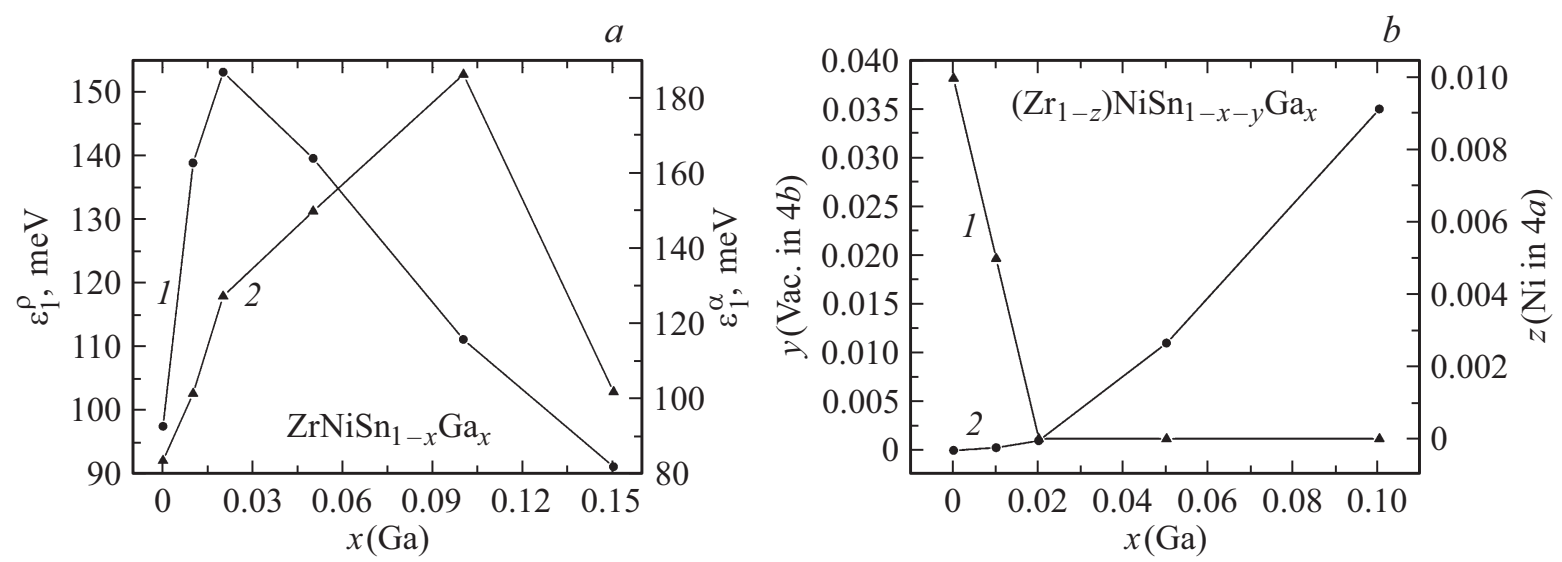

Рис. 7. Изменение значений энергий активации $\varepsilon_{1}^{\rho}(x)(1)$ и $\varepsilon_{1}^{\alpha}(x)(2)(a)$ и динамика изменения концентрации Ni $(z)$ в позиции $4 a$ атомов $\mathrm{Zr}(1)$ и вакансий $(y)$ в позиции $4 b$ атомов $\mathrm{Sn}(2)(b)$ в $\mathrm{ZrNiSn}_{1-x} \mathrm{Ga}_{x}$. 

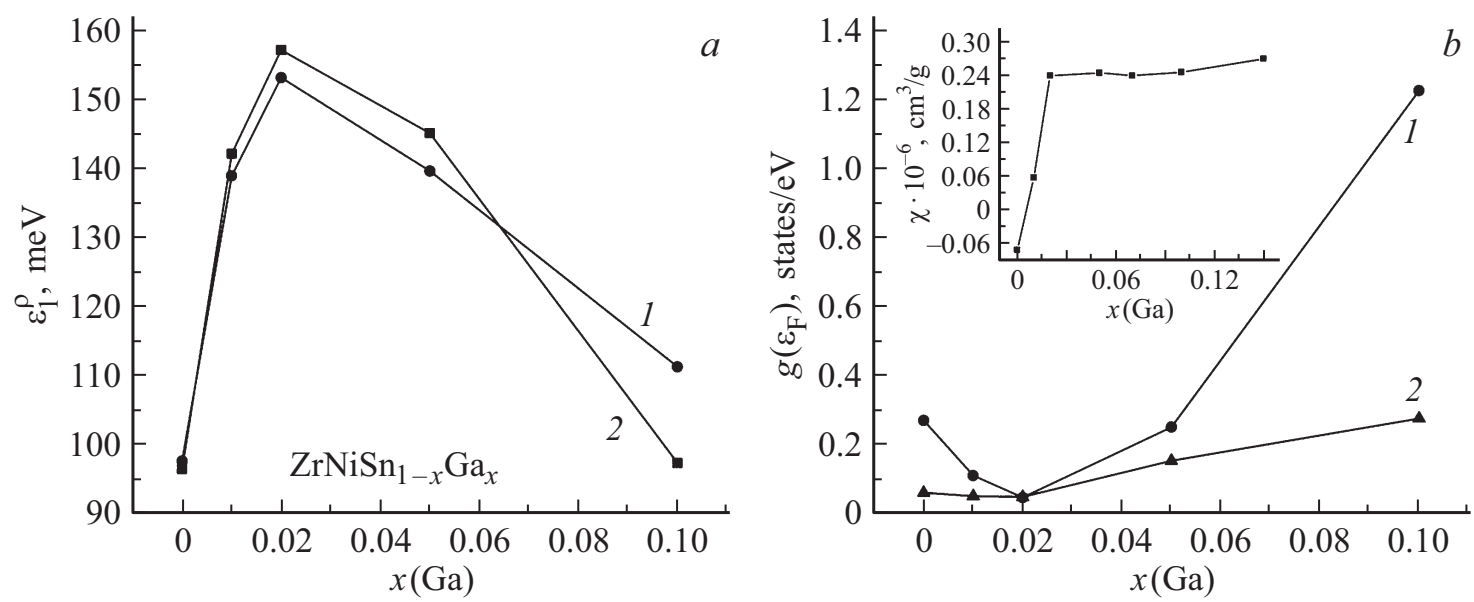

Рис. 8. Изменение значений энергии активации $\varepsilon_{1}^{\rho}(x)(a): 1-$ эксперимент, 2 - расчет с учетом генерирования донорно-акцепторных пар и плотности электронных состояний на уровне Ферми $g\left(\varepsilon_{\mathrm{F}}\right)(b) \mathrm{ZrNiSn}_{1-x} \mathrm{Ga}_{x}$ : $1-\mathrm{c}_{\text {yчетом }}$ генерирования акцепторов, 2 - с учетом генерирования донорно-акцепторных пар. На вставке $b-$ изменение значений магнитной восприимчивости $\chi(x)$.

Можно предположить, что для обеспечения устойчивости структуры $\mathrm{ZrNiSn}_{1-x} \mathrm{Ga}_{x}$ и принципа электронейтральности в позиции $4 b$ атомов $\mathrm{Sn}$ одновременно генерируются как структурные дефекты акцепторной природы, так и донорной (вакансии), концентрация которых увеличивается с ростом концентрации примеси Ga. В этом случае формулу полупроводника можем записать как $\left(\mathrm{Zr}_{1-z} \mathrm{Ni}_{z}\right) \mathrm{Ni} \mathrm{Sn}_{1-x-y} \mathrm{Ga}_{x}$, где $y-$ концентрация вакансий в позиции $4 b$ атомов $\mathrm{Sn}$.

Анализ поведения энергетических характеристик $\mathrm{ZrNiSn}_{1-x} \mathrm{Ga}_{x}$, в частности изменение значений энергии активации $\varepsilon_{1}^{\rho}(x)$ и амплитуды модуляции зон непрерывных энергий $\varepsilon_{1}^{\alpha}$, также показывает, что в полупроводнике одновременно генерируются акцепторы и доноры (рис. 7,a). Так, в $n$-ZrNiSn значение энергии $\varepsilon_{1}^{\rho}(x=0)=97.6$ мэВ показывает величину энергетической щели, отделяющую уровень Ферми $\varepsilon_{\mathrm{F}}$ от уровня протекания зоны проводимости. Легирование полупроводника $n$-типа акцепторной примесью $\mathrm{Ga}$ увеличивает степень компенсации, а уровень Ферми „углубляется“" до значений $\varepsilon_{1}^{\rho}(x=0.01)=138.8$ мэВ и $\varepsilon_{1}^{\rho}(x=0.02)=153.2$ мэВ. Учитывая, что при высоких температурах знак коэффициента термоэдс отрицательный, следует, что значения $\varepsilon_{1}^{\rho}(x=0.01)$ и $\varepsilon_{1}^{\rho}(x=0.02)$ дают глубину залегания уровня Ферми относительно уровня протекания зоны проводимости. Напомним, что значения энергии активации $\varepsilon_{1}^{\rho}(x)$ вычисляли с высокотемпературных участков зависимостей $\ln \rho(1 / T)$ (рис. 4).

Отрицательные значения коэффициента термоэдс и уменьшение значений $\varepsilon_{1}^{\rho}(x)$ при $x>0.02$ от $\varepsilon_{1}^{\rho}(x=0.05)=139.5$ мэВ до $\varepsilon_{1}^{\rho}(x=0.10)=111.2$ мэВ и $\varepsilon_{1}^{\rho}(x=0.15)=91.3$ мэВ показывают, что уровень Ферми $\varepsilon_{\mathrm{F}}$ совершил реверс и движется к уровню протекания зоны проводимости. Это значит, что в $\mathrm{ZrNiSn}_{1-x} \mathrm{Ga}_{x}$ появились электроны, а это возможно только при условии одновременного генерирования доноров $\varepsilon_{\mathrm{D}}^{2}$, концентрация которых также увеличивается с ростом концентрации примеси $\mathrm{Ga}$.

С другой стороны, одновременное генерирование в $\mathrm{ZrNiSn}_{1-x} \mathrm{Ga}_{x}$ доноров и акцепторов изменяет степень компенсации и значение амплитуды модуляции зон непрерывных энергий $[11,12]$. На рис. 7, a показано изменение значений энергии активации $\varepsilon_{1}^{\alpha}(x)$, которые пропорциональны амплитуде модуляции зон непрерывных энергий [1]. Видно, что в случае $n-\mathrm{ZrNiSn}$ амплитуда модуляции равна $\varepsilon_{1}^{\alpha}(x=0)=83.8$ мэВ. Легирование полупроводника акцепторной примесью Ga $(x=0.01)$ резко увеличивает степень компенсации, на что указывает рост значений амплитуды модуляции до $\varepsilon_{1}^{\alpha}(x=0.01)=101.6$ мэВ.

Дальнейшее увеличение концентрации акцепторов благодаря одновременному генерированию доноров увеличивает степень компенсации и амплитуду модуляции от $\varepsilon_{1}^{\alpha}(x=0.02)=127.3$ мэВ до $\varepsilon_{1}^{\alpha}(x=0.05)=$ $=149.8$ мэВ $\quad$ и $\quad \varepsilon_{1}^{\alpha}(x=0.10)=186$ мэВ. Спад зависимости $\varepsilon_{1}^{\alpha}(x)$ при концентрации $x>0.10$ может свидетельствовать о том, что число ионизированных доноров увеличивается быстрее, чем число ионизированных акцепторов. При значительных концентрациях генерированных в кристалле донорно-акцепторных пар $(x=0.15)$, когда волновые функции локализованных состояний $\varepsilon_{\mathrm{D}}^{2}$ перекрываются, при низких температурах происходит металлизация проводимости (рис. 4).

Таким образом, результаты структурных, кинетических и энергетических характеристик $\mathrm{ZrNiSn}_{1-x} \mathrm{Ga}_{x}$ указывают на сложный механизм одновременного генерирования в кристалле структурных дефектов акцепторной и донорной природы. Однако их недостаточно для идентификации самого механизма генерирования донорно-акцепторных пар. 

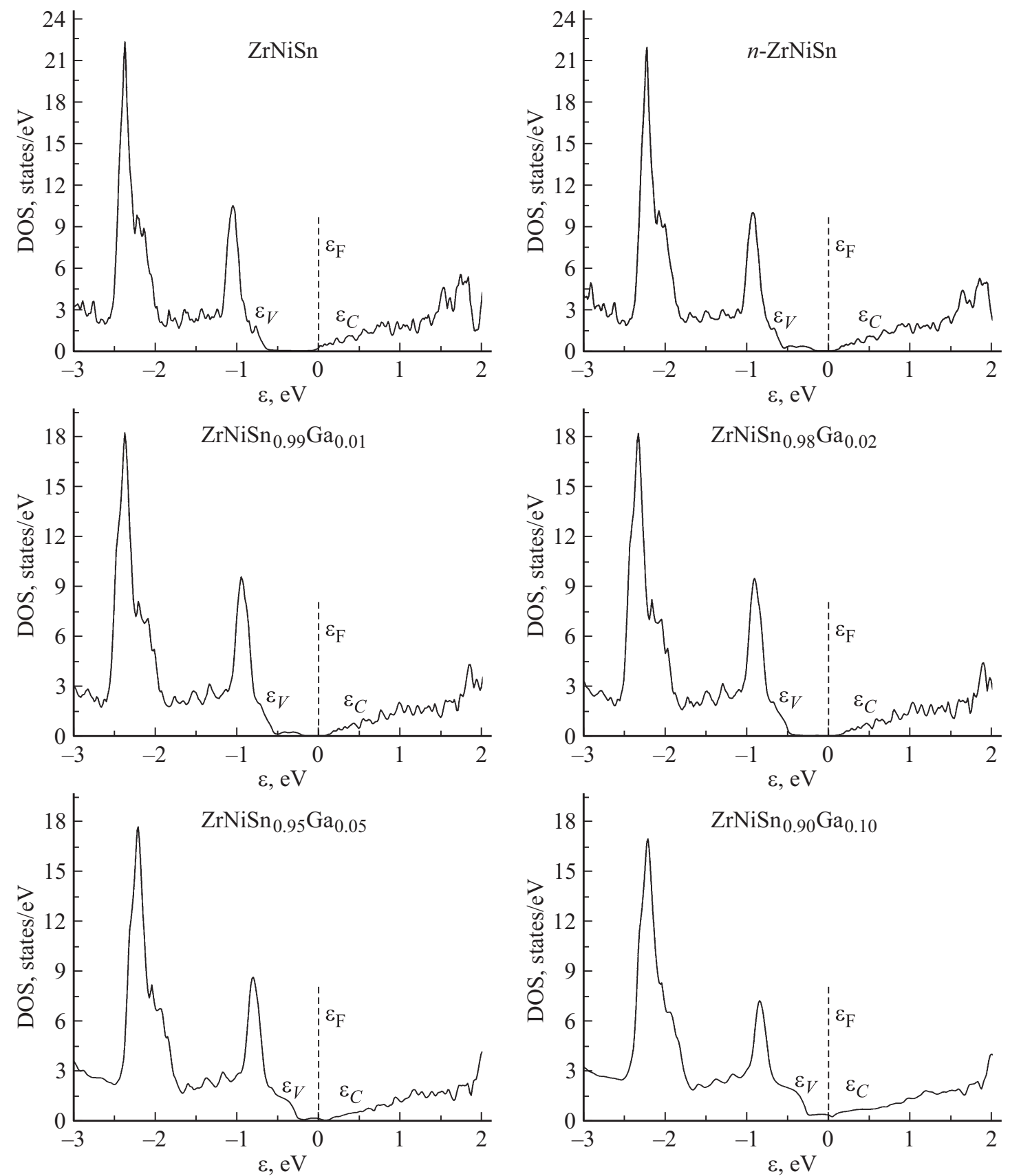

Рис. 9. Расчет изменения плотности электронных состояний DOS $\mathrm{ZrNiSn}_{1-x} \mathrm{Ga}_{x}$ с учетом генерирования структурных дефектов акцепторной и донорной природы.

\section{6. Уточнение электронной структуры $\mathrm{ZrNiSn}_{1-x} \mathrm{Ga}_{x}$}

Исходя из того, что проведенные исследования не дали информации о механизмах генерирования в $\mathrm{ZrNiSn}_{1-x} \mathrm{Ga}_{x}$ дефектов различной природы, решим обратную задачу. Известно, что для расчета энергии электрона в первой зоне Бриллюэна необходимо знать расположения атомов (или их отсутствие - вакансии) в узлах кристаллической решетки. С другой стороны, наименьшее изменение структуры кристалла изменяет его локальную симметрию и плотность электронных состояний. В таком случае адекватность результатов расчета DOS и результатов эксперимента по умолчанию предусматривает, что модель структуры вещества в полной мере отражает пространственное расположение атомов в реальной структуре кристалла, что недоступно при использовании рентгеновских методов исследования.

Обладая экспериментальными результатами скорости дрейфа уровня Ферми $\varepsilon_{\mathrm{F}} \mathrm{ZrNiSn}_{1-x} \mathrm{Ga}_{x}$ (рис. 7, $a$, кривая 1), искали степень компенсации полупроводника, которая задает скорость движения уровня Ферми, мак- 
симально близкую к экспериментальным значениям. Расчеты DOS проведены практически для всех вариантов как расположения атомов в узлах элементарной ячейки, так и степени занятости всех позиций собственными или чужими атомами, а также наличия в них вакансий. Оказалось, что наиболее приемлемым является вариант расположения атомов в упорядоченной, но деформированной структуре, в которой произошли следующие изменения:

а) кристаллическая структура $n$-ZrNiSn неупорядочена („локальная аморфизация“) из-за частичного занятия, до $\sim 1 \%(z \approx 0.01)$, атомами Ni позиции $4 a$ атомов $\mathrm{Zr}$, что приводит к генерирации в полупроводнике дефектов донорной природы (формула $\left.\left(\mathrm{Zr}_{1-z} \mathrm{Ni}_{z}\right) \mathrm{NiSn}\right)$;

б) атомы примеси $\mathrm{Ga}$ упорядочивают структуру и уменьшают число дефектов донорной природы: $\mathrm{Ni}$ покидает позицию атомов $\mathrm{Zr} \quad(4 a)$ (формула $\left.\left(\mathrm{Zr}_{1-z} \mathrm{Ni}_{z}\right) \mathrm{NiSn}_{1-x} \mathrm{Ga}_{x}\right)$;

в) занятие примесными атомами Gа кристаллографической позиции $4 b$ атомов Sn генерирует структурные дефекты акцепторной природы;

г) генерирование и увеличение числа вакансий $(y)$ в позиции $4 b$ атомов $\mathrm{Sn}$ (формула $\left.\left(\mathrm{Zr}_{1-z} \mathrm{Ni}_{z}\right) \mathrm{NiSn}_{1-x-y} \mathrm{Ga}_{x}\right)$. Физический смысл этого изменения (как результат формальных расчетов) заключается в том, что одновременное генерирование донорно-акцепторных пар обеспечивает принцип электронейтральности и устойчивость структуры термоэлектрического материала.

На рис. 7, $b$ показаны результаты расчета динамики изменения всех структурных дефектов при сильном легировании $n$ - ZrNiSn акцепторной примесью $\mathrm{Ga}$, что обеспечивает в пределах ошибки скорость движения уровня Ферми $\varepsilon_{\mathrm{F}}$ (рис. $8, a$, кривая 2 ), близкую к значению, рассчитанному из зависимостей $\ln \rho(1 / T)$ (рис. $8, a$, кривая 1).

На основании данных о пространственном расположении атомов в $\mathrm{ZrNiSn}_{1-x} \mathrm{Ga}_{x}$ проведен расчет плотности электронных состояний на уровне Ферми $g\left(\varepsilon_{\mathrm{F}}\right)$ (рис. $8, b$ ). На рис. $8, b$ для сравнения приведены результаты расчета $g\left(\varepsilon_{\mathrm{F}}\right)$ как для случая генерирования в $\mathrm{ZrNiSn}_{1-x} \mathrm{Ga}_{x}$ структурных дефектов акцепторной природы (кривая 1), так и генерирования донорно-акцепторных пар (кривая 2). Видно, что плотность электронных состояний на уровне Ферми $g\left(\varepsilon_{\mathrm{F}}\right)$ для случая генерирования в полупроводнике донорно-акцепторных пар изменяется намного медленнее, чем если бы в полупроводнике генерировались только акцепторы. Этот результат совпадает с результатами кинетических исследований $\mathrm{ZrNiSn}_{1-x} \mathrm{Ga}_{x}$.

Расчет плотности электронных состояний DOS (рис. 9) с учетом генерирования структурных дефектов акцепторной и донорной природы показывает отличия в положении уровня Ферми $\varepsilon_{\mathrm{F}}$ для упорядоченного варианта структуры $\mathrm{ZrNiSn}$, при наличии „локальной аморфизации“ в $n$-ZrNiSn, а также в образцах $\mathrm{ZrNiSn}_{1-x} \mathrm{Ga}_{x}$.
Интересными оказались результаты исследований магнитной восприимчивости $\chi \mathrm{ZrNiSn}_{1-x} \mathrm{Ga}_{x}$ (см. вставку на рис. $8, b)$, подтверждающие вывод о генерировании донорно-акцепторных пар. Оказалось, что образцы $\mathrm{ZrNiSn}_{1-x} \mathrm{Ga}_{x}(x>0.01)$ являются парамагнетиками Паули, магнитная восприимчивость $\chi$ которых определяется исключительно электронным газом и пропорциональна плотности состояний на уровне Ферми $g\left(\varepsilon_{\mathrm{F}}\right)$. При концентрации примеси $x>0.03$ зависимость $\chi(x)$ резко изменяет наклон, выходит на плато и практически не изменяется до $x=0.15$. Получается, что увеличение концентрации акцепторной примеси и возможное увеличение концентрации свободных дырок практически не изменяют значений $g\left(\varepsilon_{\mathrm{F}}\right)$. Такое поведение $\chi(x)$ $\left(\chi \sim g\left(\varepsilon_{\mathrm{F}}\right)\right)$ возможно только при условии появления в $\mathrm{ZrNiSn}_{1-x} \mathrm{Ga}_{x}$ носителей тока противоположного знака как результат генерирования донорно-акцепторных пар, что и обуславливает неизменность значений $g\left(\varepsilon_{\mathrm{F}}\right)$. Отметим, что $n$ - ZrNiSn - слабый диамагнетик, на что указывают отрицательные значения магнитной восприимчивости: $\chi(x=0)=-0.07 \mathrm{~cm}^{3} / \Gamma$, а рост зависимости $\chi(x)$ при $x=0-0.01$ не связан с увеличением плотности состояний на уровне Ферми $g\left(\varepsilon_{\mathrm{F}}\right)$.

\section{7. Заключение}

Таким образом, в результате комплексного исследования кристаллической и электронной структур, кинетических и магнитных характеристик полупроводникового термоэлектрического материала $\mathrm{ZrNiSn}_{1-x} \mathrm{Ga}_{x}$ установлен механизм одновременного генерирования в кристаллографической позиции $4 b$ атомов $\mathrm{Sn}$ структурных дефектов акцепторной и донорной природы (донорноакцепторные пары), которые изменяют степень компенсации и определяют механизм проводимости полупроводника. Исследуемый материал обладает упорядоченной кристаллической структурой, что обеспечит стабильность его характеристик.

\section{Список литературы}

[1] В.А. Ромака, В.В. Ромака, Ю.В. Стаднык. Интерметаллические полупроводники: свойства и применения (Львов, Львовская политехника, 2011).

[2] M. Gürth, G. Rogl, V.V. Romaka, E. Bauer, P. Rogl. Acta Materialia, 104, 210 (2016).

[3] M. Schwall, B. Balke. Phys. Chem. Chem. Phys., 15 (6), 1868 (2013).

[4] D. Fruchart, V.A. Romaka, Yu.V. Stadnyk, L.P. Romaka, Yu.K. Gorelenko, M.G. Shelyapina, V.F. Chekurin. J. Alloys Comp., 438, 8 (2007).

[5] В.А. Ромака, М.Г. Шеляпина, Ю.К. Гореленко, D. Fruchart, Ю.В. Стаднык, Л.П. Ромака, В.Ф. Чекурин. ФТП, 40 (6), 676 (2006).

[6] В.А. Ромака, Ю.В. Стаднык, D. Fruchart, Л.П. Ромака, А.М. Горынь, Ю.К. Гореленко, Т.И. Доминюк. ФТП, 43 (3), 297 (2009). 
[7] В.А. Ромака, E.K. Hlil, Я.В. Сколоздра, Р. Rogl, Ю.В. Стаднык, Л.П. Ромака, А.М. Горынь. ФТП, 43 (9), 1157 (2009).

[8] V.A. Romaka, P.-F. Rogl, L.P. Romaka, Yu.V. Stadnyk, V.Ya. Krayovsky, D. Kaczorowski, A.M. Horyn. J. Thermoelectricity, № 3, 24 (2016).

[9] Н. Мотт, Т. Дэвис. Электронные процессы в некристаллических веществах (М., Мир, 1982) [Пер. с англ.: N.F. Mott, E.A. Davis. Electron processes in non-crystalline materials (Oxford, Clarendon Press, 1979)].

[10] Б.И. Шкловский, А.Л. Эфрос. Электронные свойства легированных полупроводников (М., Наука, 1979).

[11] Б.И. Шкловский, А.Л. Эфрос. ЖЭТФ, 61 (2), 816 (1971).

[12] Б.И. Шкловский, А.Л. Эфрос. ЖЭТФ, 62 (3), 1156 (1972).

[13] T. Roisnel, J. Rodriguez-Carvajal. Mater. Sci. Forum, Proc. EPDIC7, 378-381, 118 (2001).

[14] M. Schroter, H. Ebert, H. Akai, P. Entel, E. Hoffmann, G.G. Reddy. Phys. Rev. B, 52, 188 (1995).

[15] V.L. Moruzzi, J.F. Janak, A.R. Williams. Calculated electronic properties of metals (N. Y., Pergamon Press, 1978).

[16] В.В. Ромака, Л.П. Ромака, В.Я. Крайовский, Ю.В. Стаднык. Станиды редкоземельных и переходных металлов (Львов, Львовская политехника, 2015).

[17] V.V. Romaka, P. Rogl, L. Romaka, Yu. Stadnyk, A. Grytsiv, O. Lakh, V. Krayovsky. Intermetallics, 35, 45 (2013).

Редактор А.Н. Смирнов

\title{
The mechanism of generation of donor-acceptor pairs in heavily doped $n$-ZrNiSn with $\mathrm{Ga}$ acceptor impurity
}

\author{
V.A. Romaka ${ }^{1,2}$, P.-F. Rog/ ${ }^{3}$, D. Frushart ${ }^{4}$, \\ D. Kaczorowski ${ }^{5}$
}

${ }^{1}$ Ya. Pidstryhach Institute for Applied Problems of Mechanics and Mathematics National Academy of Sciences of Ukraine,

79060 Lviv, Ukraine

${ }^{2}$ National University „Lvivska Politechnika“, 79013 Lviv, Ukraine

${ }^{3}$ Institut fur Physikalische Chemie, Universitet Wien,

A-1090 Wien, Austria

${ }^{4}$ Institut Néel, CNRS, BP 166 ,

38042 Grenoble Cedex 9, France

${ }^{5}$ Trzebiatowski Institute

of Low Temperature and Structure Research,

Polish Academy of Sciences,

50-950 Wroclaw, Poland

Abstract The mechanism of simultaneous generation of donoracceptor pairs in heavily doped $n$-ZrNiSn with Ga acceptor impurity is established. The modeled distribution of atoms in the crystal lattice of $\mathrm{ZrNiSn}_{1-x} \mathrm{Ga}_{x}$ showed that the speed of movement of Fermi level $\varepsilon_{\mathrm{F}}$, obtained from the band structure calculations is in agreement with experimental extracted from $\ln \rho(1 / T)$ dependencies. It is shown that with substitution of $\mathrm{Sn}$ $\left(5 s^{2} 5 p^{2}\right)$ with $\mathrm{Ga}\left(4 s^{2} 4 p^{1}\right)$ atoms in $4 b$ crystallographic site both acceptor and donor (vacancies in $4 b$ site) defects are generated. The results are discussed within the Shklovskiy-Efros model of heavily doped and compensated semiconductor. 